\title{
Mobile EEG on the bike: disentangling attentional and physical contributions to auditory attention tasks.
}

\author{
Rob Zink ${ }^{1,2}$, Borbála Hunyadi ${ }^{1,2}$, Sabine Van Huffel ${ }^{1,2}$, \\ Maarten De $\operatorname{Vos}^{3}$ \\ ${ }^{1}$ KU Leuven, Department of Electrical Engineering (ESAT), STADIUS Center \\ for Dynamical Systems, Signal Processing and Data Analytics, Kasteelpark \\ Arenberg 10, 3001 Leuven, Belgium. \\ ${ }^{2}$ iMinds Medical IT, Leuven, Belgium. \\ ${ }^{3}$ Engineering Department, Oxford University, Oxford, United Kingdom. \\ E-mail: rob.zink@esat.kuleuven.be
}

\begin{abstract}
Objective In the past few years there has been a growing interest in studying brain functioning in natural, real-life situations. Mobile EEG allows to study the brain in real unconstrained environments but it faces the intrinsic challenge that it is impossible to disentangle observed changes in brain activity due to increase in cognitive demands by the complex natural environment or due to the physical involvement. In this work we aim to disentangle the influence of cognitive demands and distractions that arise from such outdoor unconstrained recordings. Approach We evaluate the ERP and single trial characteristics of a three-class auditory oddball paradigm recorded in outdoor scenario's while peddling on a fixed bike or biking freely around. In addition we also carefully evaluate the trial specific motion artifacts through independent gyro measurements and control for muscle artifacts. Main results A decrease in P300 amplitude was observed in the free biking condition as compared to the fixed bike conditions. Above chance P300 single-trial classification in highly dynamic real life environments while biking outdoors was achieved. Certain significant artifact patterns were identified in the free biking condition, but neither these nor the increase in movement (as derived from continuous gyrometer measurements) can explain the differences in classification accuracy and P300 waveform differences with full clarity. The increased cognitive load in real-life scenarios is shown to play a major role in the observed differences. Significance Our findings suggest that auditory oddball results measured in natural real-life scenarios are influenced mainly by increased cognitive load due to being in an unconstrained environment.
\end{abstract}

Keywords: Mobile EEG, Auditory Attention, Cycling, P300, Gyroscope, BrainComputer-Interface, Real-life Environment. 


\section{Introduction}

The most studied feature in Brain-computer-interfaces (BCI) with non-invasive electroencephalography (EEG) is the P300, generated in response to rare and taskrelevant stimuli (e.g. $[1,2,3,4]$ ). Originally, BCIs were proposed to provide accessibility to computers for locked-in patients [5]. More recently, they have also gained potential in applications for healthy users $[6,7]$; especially when they are not bound to highly restricted traditional laboratory settings. In the past few years there has been a growing interest in studies that utilize mobile EEG in natural, reallife situations $[8,9,10,11,12,13,14]$. The success of these studies is closely related to the data quality observed with miniaturized portable EEG hardware such as Emotiv (www.emotiv.com) and SMARTING (www.mBrainTrain.com). For example the original Emotiv hardware was found to be inferior to a medical grade system $[15,16]$, however a modified setup allowed high quality recordings [8]. Moreover, it was recently demonstrated that such mobile devices can deliver comparable waveforms as traditional EEG systems [17]. The presence of mobile EEG creates new possibilities for investigating cognitive mechanisms in natural environments in contrast to limited and artificial laboratory setups [6, 7, 18]. Although real-life scenarios have been mimicked in labs [19, 20, 21, 22, 23], true real-life EEG recordings are sparse (e.g. $[8,9,24,25,26])$. These studies demonstrated the feasibility of investigating auditory attention while walking which mimics closely a practical application of auditory BCI in real-life [10, 11]. However, these studies face the challenge that the observed differences (e.g. decreased P300 while walking as compared to still conditions $[8,9]$ ) can be attributed to either the higher cognitive demands or to the contributions of physical activity in such auditory attention tasks.

In mobile real-life experiments the users mental focus is less controllable compared to indoor recordings. Additionally, unconstrained setups may induce additional artifacts such as head and body motions or increased muscle activity [27, 28]. Walking outdoors while performing an auditory oddball task leads to significantly lower classification accuracies compared to sitting still indoors $[8,9]$. De Vos et al. speculated that the subjects in the walking condition endured increased cognitive demands and distractions that caused the lower performance. In the current study subjects were presented with the same three-class auditory oddball paradigm in three different outdoor conditions to disentangle effects of physical activity from higher cognitive demand. In one condition subjects sat still on a regular bike fixed in a framework so that they did not move. Secondly subjects sat on the fixed bike and additionally pedaled at a user chosen comfortable pace.
The third condition comprised of biking freely on a preexplored course on the university campus. This was the most demanding condition as the subjects need to attend to the auditory task, execute motor patterns to pedal and deal with the complex environment as they move around the streets. The pedaling condition induces muscle and motor activity but probably no increased cognitive load as compared to actual biking around campus. Sitting completely still in a bike position can be seen as the most restricted condition (control) in which only environmental noise could distract the subjects. All conditions took place outside to ensure similar degrees of random environmental effects (i.e. weather conditions, cars passing by, people talking in the background).

Several EEG laboratory studies investigated specifically the effect of cycling exercise on the P300. Studies have reported an increase in P300 amplitude during or after physical pedaling on an exercise bike in response to visual attention tasks $[29,30,31]$. Vogt et al. contrastively reported no influence of exercise on the P300 in a virtual reality study [32]. Furthermore, Yagi et al. described decreased P300 amplitudes in during exercise [33]. Potential reason for the decrease in P300 in these studies is reasoned to be the increase in cognitive load that diminishes the P300 due to lower attentional resources. Yet another study indicated increases in P300 only during 72 to 108 min of exercise [34]. These studies did not evaluate the fluctuations in P300 in a true real-life enviroment. Given that in our setup the cognitive demands will be higher as compared to the aforemented lab-setups we would hypothesize for a decrease in P300 in the real biking conditions as compared to the fixed bike conditions. Additionally, very few mobile EEG studies explicitly controlled for the amount of movement while recording in real-life situations, although many systems allow to simultaneously record the level of movement. Lab studies usually try to minimize head and body motion and the influence of physical motion on, for example, the P300 is avoided principally by trial rejection. In the current setup we have instantaneous correlates of head movement from a 3D gyro measurement that allows to investigate these potential artifacts and derive measures to compare the motion to ERP and classification features. Being in less constrained scenarios, subjects will be more mobile, leading to notable EMG artifacts in the EEG (e.g. increased muscle activity to keep head up) [35, 36, 37, 38, 39]. For an overview on the methodological challenges of recording EEG during motion we refer the reader to [38]. Past experience has shown that CCA-based demixing of the data leads to a superior removal of artifacts as compared to the wide-spread ICA-based demixing [40, 41]. Here we follow this approach to 
extract muscle related sources from the EEG.

To summarize, the main goal is to evaluate the influence of cognitive distraction and physical engagement on auditory attention correlates in unconstrained out-of-the-lab scenarios. We will investigate both ERP and single trial correlates recorded from subjects seated on a bike without pedaling, with pedaling and while biking in real life environments. Secondly, we will investigate the effect of different amounts of movement, as quantified with a 3D gyrometer signal on P300 features. Finally we compare the main P300 waveform results before and after correcting for EMG activity.

The article is structured as follows: Firstly, we provide a brief overview of the stimuli, hardware, procedure and data acquisition with the mobile EEG. Secondly, we discuss the data analysis of the derived ERP features and classification methods used. Thirdly, the obtained results are presented in terms of ERP results, motion quantification and classifier output. Fourthly, the influence of EMG artifact removal on the aforementioned results are presented. Finally, we discuss the effect of real-life circumstances on auditory attention and the complementary information obtained from physical motion measures followed by future perspectives for the field of mobile EEG studies.

\section{Methods}

\subsection{Participants}

Fifteen subjects (mean age (SD): 27.1 (2.5), four women) participated in the current trial. Subjects reported normal hearing and no past or present neurological or psychiatric conditions. All participants signed informed consent prior to participation. The ethics committee of the KU Leuven approved the experimental setup. All subjects reported to be able to ride a bike confidently.

\subsection{Stimuli and procedure}

A three-class oddball auditory task was used as proposed by [4]. A standard tone $(900 \mathrm{~Hz})$ and two deviant tones $(600 \mathrm{~Hz}, 1200 \mathrm{~Hz})$ of $62 \mathrm{~ms}$ duration (10ms rise/fall time) were presented binaurally through low-cost consumer headphones (Sennheizer mx460) in random order. A mean inter-stimulus interval of 1000 ms (jitter [0-375] ms) was used. The participants task was to silently count the target tones and ignore the two other tones. Eight subjects attended the lower $(600 \mathrm{~Hz})$ tone and the others attended the high $(1200 \mathrm{~Hz})$ tone. The subjects' attention to a certain tone should elicit a distinguishable P300 (target) as compared to the non-attended tones (non-Targets). 504 standards, 92 non-target deviants and target deviants were presented in the first condition. In the second recording a few more trials were presented in order to create a valid task silently counting targets (105 stimuli). In the third recording the stimulus numbers were equal to the first condition. Due to technical issues, we recorded only 69 trials in 2 subjects. In all other subjects and conditions the trials were truncated, restricting each condition to 92 trials in order to ensure equal comparisons between conditions. The recordings took place outdoors on the campus of the KU Leuven. The subjects performed one recording while sitting still on a bike in a fixed standard facing nature and one recording while pedaling on the bike in the fixed standard. The third condition involved biking freely around on a 500m course on campus for the period of the experiment. An overview of the three conditions is presented in Figure 1. The biking route (move condition) was predefined and practiced before the start of the experiment. The order of the conditions was balanced between subjects. These three conditions will be referred to as Still, Pedal and Move from here on. During the experiment, participants carried an ultrabook laptop (weight $<2 \mathrm{~kg}$ ) in a backpack. Each condition lasted roughly 12 minutes. For the Pedal and Move conditions subjects were free to pedal at a comfortable pace. On average, subjects biked 12 $\mathrm{km} / \mathrm{h}$, as derived from the average time taken to bike once around the course. The course for biking in the Move condition involved regular streets with traffic (i.e. mostly pedestrians and cyclists, very few cars). Note that during all recordings, subjects were outdoors exposed to naturally occuring (sound) distraction (e.g. insects which seemed to be attracted to the gel, wind, car traffic noise and people passing by). Recordings took place during spring and summer of 2015; the temperature varied between 10 and 30 degrees Celsius. Recordings with strong wind $>4 \mathrm{Bft}$ and precipitation were avoided. OpenViBE software [42] running on the ultrabook was used for stimulus delivery and experimental control. After the experiment, subjects were asked to rate (on a scale from 1 to 10 ) the three conditions according to the degree they enjoyed each of the recordings. This was included in order to obtain a coarse measure of motivation as this has been shown to influence the P300 waveform [43].

\subsection{Data acquisition}

The acquisition was conducted with a SMARTING mobile EEG amplifier from mBrainTrain (Belgrade, Serbia, www.mbraintrain.com). This comprises a wireless EEG system running on a notebook using a small 24-channel amplifier with similar characteristics to a stationary laboratory amplifier $(<1 \mathrm{~V}$ peak to peak noise; $500 \mathrm{~Hz}$ sampling rate). The EEG was measured using $24 \mathrm{Ag} / \mathrm{AgCl}$ passive scalp electrodes (Easycap, www.easycap.com), placed according to the 

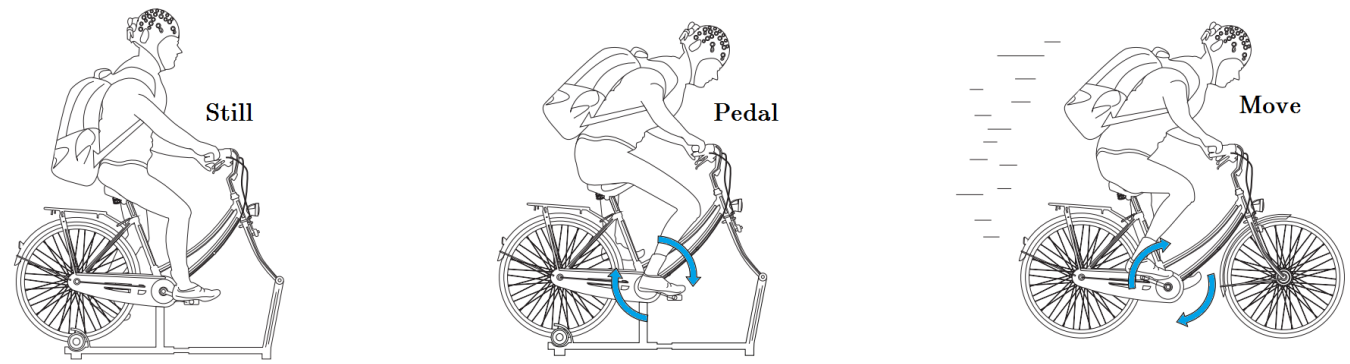

Figure 1. Schematic of the Still, Pedal and Move conditions. Note that in the Pedal conditions subjects sit on the fixed bike like the Still condition and pedal like they were biking in the Move condition. All recordings took place outdoors at or next to roads on the KU Leuven campus

10-20 standard system with positions: FP1, FP2, $\mathrm{Fz}, \mathrm{F} 7, \mathrm{~F} 8, \mathrm{FC} 1, \mathrm{FC} 2, \mathrm{Cz}, \mathrm{C} 3, \mathrm{C} 4, \mathrm{~T} 7, \mathrm{~T} 8, \mathrm{CPz}$ CP1, CP2, CP5, CP6, TP9, TP10, Pz, P3, P4, $\mathrm{O} 1$ and $\mathrm{O} 2$. Impedances were kept below $10 \mathrm{kOhm}$ and an abrasive electrolyte-gel was applied in each electrode. In addition three-axis (x,y,z) gyroscope data was measured at similar sampling rate as the EEG. The gyroscope is included in the amplifier which is placed at the back of the head. Data were recorded through Openvibe [42] and analyzed offline using custom made Matlab scripts, EEGLAB13.4.3b [44] and BCILAB1.1 [45].

\subsection{Data analysis}

2.4.1. Preprocessing Extended Infomax ICA was used to remove EOG activity by means of manual component selection that represented eye-blinks and lateral eye movements. As a pre-processing step for ICA all EEG data was high-pass filtered at $1 \mathrm{~Hz}$ as this is shown to produce stable ICA results for artifact removal [46]. Consecutively the learned weights of the marked EOG components were applied to a common band-pass filtered [0.5-20] Hz dataset. Signals were rereferenced offline to the mean of TP9 and TP10 and epochs were extracted $200 \mathrm{~ms}$ before stimulus onset to $800 \mathrm{~ms}$ after. The Tp9 and Tp10 channels were removed after re-referencing. A baseline subtraction was applied based on the $[-200-0] \mathrm{ms}$ interval. The gyro-signals were high-pass filtered at $0.5 \mathrm{~Hz}$ as well. Average ERPs were calculated at the $\mathrm{Pz}$ electrode as this is the most prominent site for the P300 waveform. Visual representation of the ERPs was achieved after removing artifactual single-trials as implemented in EEGLAB [44]. Rejection criteria were based on abnormally distributed data and improbable data. The cutoff was defined as four standard deviations of the mean kurtosis value and probability distribution respectively. Note, all the analyses were based on the full dataset.

In addition we removed EMG activity by means of BSS-CCA. This assumes that EMG sources have significantly lower autocorrelation as compared to brain sources [40]. Removal of the EMG was achieved through BSS-CCA on 10s non-overlapping windows. After extracting 24 components (per window), the power spectral density of each source was computed. Sources with a power ratio smaller than 2 between brain band (i.e. $3-15 \mathrm{~Hz}$ ) and muscle band (i.e. 25$50 \mathrm{~Hz}$ ) were removed. This approach is similar as presented in $[40,41]$.

2.4.2. Classification For single-trial P300 classification we extracted one feature set which consisted of two different types of features: The first type comprised seventeen $47 \mathrm{~ms}$ data bins on all 22 electrodes between [0-800]ms, which is similar to previously reported work $[8,9]$ resulting in 374 features. In addition we averaged all standard-stimulus trials of the non-test subjects to create an average template. This was shown to provide an accurate estimation of the non-Target waveforms and therefore the distance to this template can be used as discriminative feature to distinguish target from non-targets trials. We down-sampled the EEG data and template to $30 \mathrm{~Hz}$ and subtracted per trial the EEG data from the template in eleven points in a 200-533ms window on all 22 electrodes, resulting in another 242 features. This feature extraction follows the approach used in previous publications [14, 47]. Both feature types were concatenated creating a total of 616 features per single-trial. We classify the EEG signals using a Linear Discriminant Analysis as it is among the most used classifiers for the P300 [48, 49, 50]. Shrinkage regularization as implemented in BCILAB [45] is used for the rLDA classification. Per subject and session the classifiers are trained and evaluated based on a five-fold cross-validation procedure. Finally, to evaluate differences between conditions further, we train per subject an rLDA classifier on data from one of the other two conditions of that same subject. For example train on the Still condition and Test on the Pedal condition (Cross-session).

2.4.3. ERP features In order to compare the ERP features we computed the maximum P300 at Pz for the 

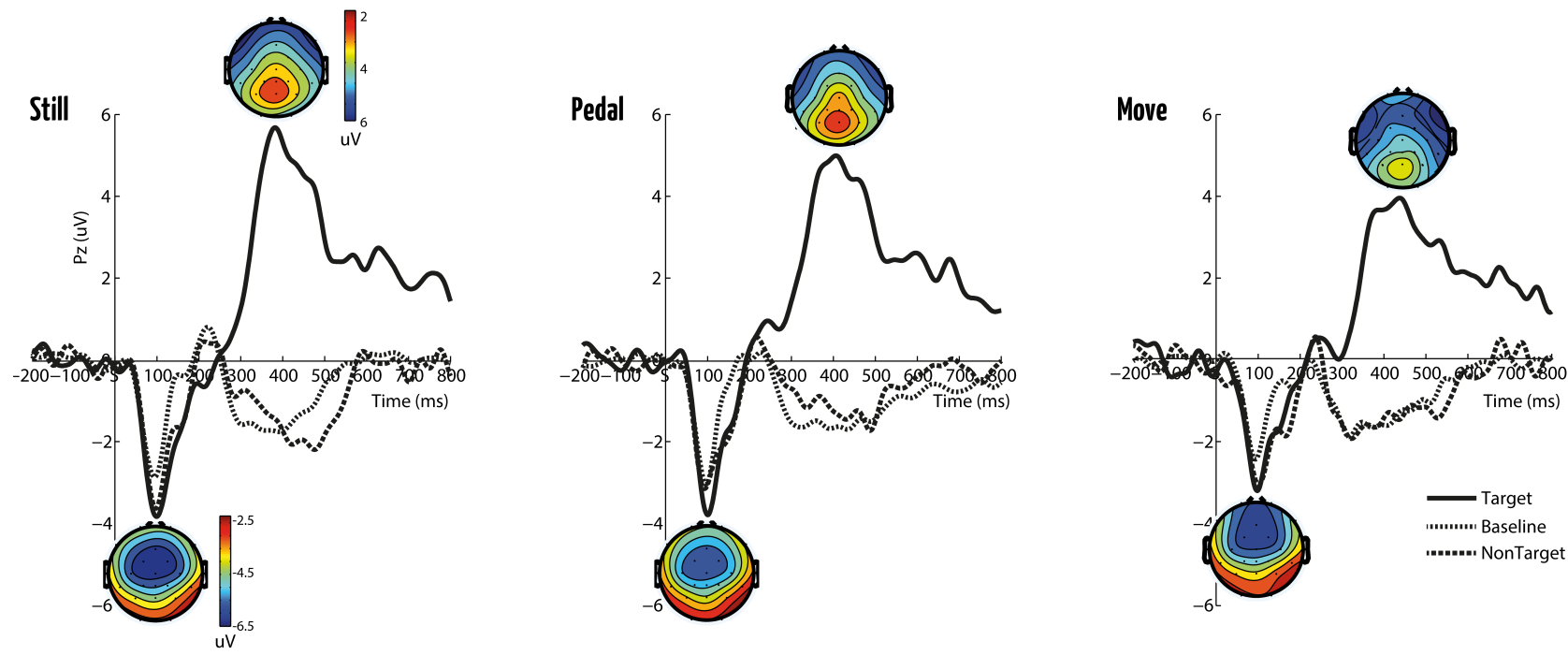

Figure 2. Grand average ERPs to the Target, nonTarget and Baseline stimuli at electrode Pz for the Still, Pedal and Move conditions from left to right respectively. At the peak N100 and P300 ERP a topoplots indicates the distribution of the peak along all electrodes for the Target stimuli. Note: for illustrational purposes artifactual single-trials were removed before plotting. Reported statistics in the text are based on the full dataset.

Target and Distractor tones for each subject in a $[200-$ 600]ms interval. Likewise we computed the minimum $\mathrm{N} 100$ at $\mathrm{Pz}$ for all tones in the $[0-200] \mathrm{ms}$ interval. This was achieved by averaging $22 \mathrm{~ms}$ (i.e. 11 samples $[-5$ $+5])$ around the peak latency value at each electrode. The root mean square (RMS) in the [-200-0]ms baseline interval was used as noise measure for the EEG which is similar to [9]. Consecutively we took the average RMS over the three gyro axes in the $[0-800] \mathrm{ms}$ interval as measure of the amount of head motion per trial. Alpha band activity $(8-12 \mathrm{~Hz})$ was calculated at electrode $\mathrm{O} 2$ as the average normalized power in this range for the Target trials.

2.4.4. Statistical analysis One-way ANOVAs were performed to determine the significance of mean group differences between the three conditions. This was used for comparing EEG RMS, N100 amplitude, Gyro RMS and classification accuracies between conditions. For the P300 comparison a repeated measures analysis of variance ANOVA was conducted with the factors recording (Still, Pedal and Move) and tone (Target, NonTarget). This way we evaluate the elicted P300 differences between the target and distractor tone and explore impact of the recording condition on this effect. In all analyses, the significance level was set at .05. Pearsons correlation coefficient is used to identify linear relationships between our derived ERP and RMS features and classification results.

\section{Results}

The ERP, Noise level, Classification and Gyro results presented here are obtained on the data without explicit control for muscle artifacts. Consequently, Section 3.5 displays the results after removal of muscle artifacts.

\section{1. $E R P$}

The average ERPs of the Baseline, Target and NonTarget tones are displayed in Figure 2 with topographies of the peak P300 and N100 for the Still, Pedal and Move condition. In all conditions a clear posterior focus of the P300 topography is visible whereas the N100 is more central. P300 amplitude in the Move condition was on average $31 \%$ and $26 \%$ lower as compared to the Still and pedal condition respectively. The repeated measures ANOVA recording (Still, Pedal, Move) $\times$ tone (Target, NonTarget) revealed a nearly significant main effect for recording, $\mathrm{F}(2,14)=2.89, \mathrm{p}=.06$, reflecting larger amplitudes for the still $($ mean $=6.8 \mu \mathrm{V})$ and pedal $($ mean $=6.3 \mu \mathrm{V})$ compared to the Move condition $($ mean $=4.7 \mu \mathrm{V})$. In addition, a significant main effect for tone emerged, $\mathrm{F}(2,14)=68.41, \mathrm{p}<.001$, confirming larger P300 amplitudes for target tones (mean $=5.96$ $\mu \mathrm{V})$ compared to distractor tones $($ mean $=1.57 \mu \mathrm{V})$. The interaction recording tone was far from reaching significance, $\mathrm{F}(2,14)=.58, \mathrm{p}=.56$.

Contrastingly the peak N100 values did not differ significantly i.e. one way ANOVA $(\mathrm{F}=0.42, \mathrm{p}=.66)$, $(\mathrm{F}=0.21, \mathrm{p}=.83)$ and $(\mathrm{F}=1.20, \mathrm{p}=.31)$ across conditions for the target, nontargets and baseline 
stimuli respectively. A slightly higher P300 latency was observed in the Move condition; 445ms $( \pm 95)$ as compared to the Still $400 \mathrm{~ms}( \pm 56)$ and pedal $388 \mathrm{~ms}$ $( \pm 89)$ latencies but this difference was not statistically significant $(\mathrm{F}=2.00, \mathrm{p}=.15)$. Correlations between the subject average P300 across the still-pedal conditions was moderate $(\mathrm{r}=.70, \mathrm{p}<.001)$, for the pedalmove $(\mathrm{r}=.61, \mathrm{p}<.001)$ and still-move $(\mathrm{r}=.55, \mathrm{p}$ $<.001$ ). Overall, subjects which had more consistent P300 waveform across conditions also had a higher P300 amplitude $(\mathrm{r}=.74, \mathrm{p}<.01)$.

\subsection{Noise levels}

The mean RMS values of the target trials' baseline at $\mathrm{Pz}$ did not show significant differences overall between conditions $(\mathrm{F}=0.14, \mathrm{p}=.87)$. These RMS values at $\mathrm{Pz}$ are similar to the ones reported during Sitting and Walking in a previous study [9]. However, at different electrode sites differences between conditions can be observed. Figure 3 illustrates the median RMS of the pre-stimulus window for all channels in the Still, Pedal and Move condition. The Pedal condition RMS values were only slightly elevated as compared to the Still condition and the RMS topography does not change. In the Move conditions on the outer electrode sites clear differences can be observed as compared to the other two conditions. This was confirmed by (marginally) significant ANOVA results on channels: FP1 $(\mathrm{F}=3.09, \mathrm{p}=.06), \mathrm{FP} 2(\mathrm{~F}=3.25$, $\mathrm{p}$ $<.05), \mathrm{O} 1(\mathrm{~F}=2.70, \mathrm{p}=.08)$ and $\mathrm{O} 2(\mathrm{~F}=4.50, \mathrm{p}<.05)$. Pairwise comparisons showed a significant increase in RMS in the Move condition as compared to either the Still or Pedal condition. The artifactual pattern in the Move condition is similar to those presented by motion artifact studies that report increased artifacts especially at the outer electrode sites [28].
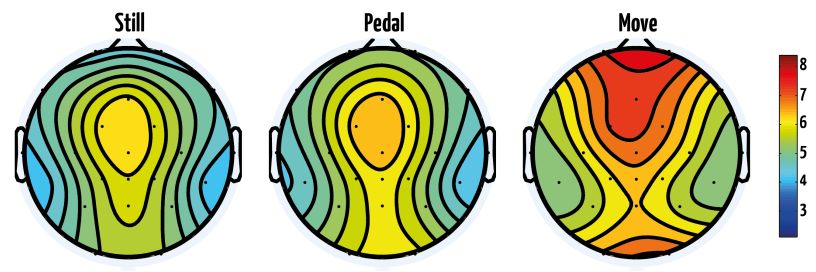

Figure 3. Median RMS values of the baseline interval [-200$0] \mathrm{ms}$ of the Target trials at each electrode for the Still, Pedal and Move condition.

\subsection{Classification}

Grand average accuracies $( \pm \mathrm{SD})$ on a single trial level are $76.6( \pm 7.0), 72.7( \pm 9.3)$ and $67.6 \%( \pm 8.5)$ for the Still, Pedal and Move condition respectively which are presented in table 1 . These differences were deemed significant $(\mathrm{F}=4.45, \mathrm{p}<.05)$. The accuracies in the Move condition were significantly lower as compared to the Still $(\mathrm{t} 14=3.89, \mathrm{p}<.01)$ and Pedal $(\mathrm{t} 14=3.31, \mathrm{p}<.01)$ condition. The difference between Pedal and Still conditions was marginally significant $(\mathrm{t} 14=2.06, \mathrm{p}=.06) . \quad$ P300 ERP amplitudes were significantly correlated to the rLDA accuracy across subjects for all three conditions; still $(\mathrm{r}=.71, \mathrm{p}<.001)$, pedal $(\mathrm{r}=.80, \mathrm{p}<.0001)$ and move $(\mathrm{r}=.64, \mathrm{p}$ $<.05)$. This indicates that individuals with larger P300 ERPs had better classification accuracies. Similarly, correlations of the single-trial classification accuracies between the still-pedal $(\mathrm{r}=.61, \mathrm{p}<0.05)$ and pedalmove $(\mathrm{r}=.78, \mathrm{p}<.001)$ were observed. This was not the case for the still-move comparison of accuracies ( $\mathrm{r}$ $=.33, \mathrm{p}=.22$ ).

Table 1. Grand-Average Session Specific accuracies ( \pm SD).

\begin{tabular}{cccc}
\hline & Still & Pedal & Move \\
\hline Accuracy & $76.6 \%( \pm 7.0)$ & $72.7 \%( \pm 9.3)$ & $67.6 \%( \pm 8.5)$ \\
\hline
\end{tabular}

Classifying across sessions lead to accuracies of $71.4( \pm 8.9)$ and $68.5( \pm 7.6)$ for the Still condition when trained on the Pedal and Move data respectively. Accuracies in the Pedal condition were $71.3( \pm 8.7)$ when trained on the Still recordings and 67.8\% ( \pm 9.4) for training on the Move condition. Finally the Move condition accuracies were $66.4( \pm 6.5)$ and $65.1( \pm 9.3)$ when trained on the Still or Pedal condition respectively. The accuracies of all cross session comparisons are summarized in Table 2.

Table 2. Grand-Average Cross-Session accuracies $( \pm \mathrm{SD})$.

\begin{tabular}{cccc}
\hline Train On & Test Still & Test Pedal & Test Move \\
\hline Still & $\mathrm{n} / \mathrm{a}$ & $71.3 \%( \pm 8.7)$ & $66.4 \%( \pm 6.5)$ \\
Pedal & $71.4 \%( \pm 8.9)$ & $\mathrm{n} / \mathrm{a}$ & $65.1 \%( \pm 9.3)$ \\
Move & $68.5 \%( \pm 7.6)$ & $67.8 \%( \pm 9.4)$ & $\mathrm{n} / \mathrm{a}$ \\
\hline
\end{tabular}

\subsection{Gyro Signals}

Average $( \pm \mathrm{SD})$ RMS values of the 3 -axis gyroscope signals are $2.4( \pm 0.6)$ in the Still, $17.4( \pm 9.7)$ in the Pedal and $27.6( \pm 8.0)$ in the Move conditions. The single-trial motion distributions are displayed in Figure 4a. As expected the mean gyro motion RMS variables were higher in the Move condition as opposed to the Pedal and Still condition. Similarly the Pedal condition gyro RMS was higher compared to the Still condition. To examine the effect of the motion in the (real-life) Move condition as measured with the gyro to the ERPs, we split per subject the trials into eight bins of low to high motion of equal trial numbers. The gyro effect on the eight grand average ERPs is depicted in Figure 4b. The color coding indicates the 
eight different levels of motion. Finally in Figure 4c the peak P300 as seen in Figure 4b is plotted in order to reveal any effect of motion level on the P300 amplitude. No decrease in P300 amplitude can be observed and these results suggest that there is no dependence of gyro motion on the ERP at Pz. Similar results were obtained by analysing the P300 waveforms on the gyro axes separately (not presented here). Finally, we investigated the relationship of the gyro motion on the accuracies as computed with the subject specific rLDA on subject level. The gyro motion level displays a moderate negative correlation to the rLDA accuracies for the Still $(\mathrm{r}=-.39, \mathrm{p}=.15)$, Pedal $(\mathrm{r}=-.51, \mathrm{p}=$ $.05)$ and Move $(\mathrm{r}=-.33, \mathrm{p}=.23)$ conditions and lower correlations to the average P300 amplitude $(\mathrm{r}=-.36$, $\mathrm{p}=.10),(\mathrm{r}=-.43, \mathrm{p}=.11)$ and $(\mathrm{r}=-.16, \mathrm{p}=.58)$ for the Still, Pedal and Move condition respectively.

\subsection{Muscle Artifacts}

After removing muscle activity, the mean RMS values of the target trials' baseline at $\mathrm{Pz}$ showed no significant differences between conditions $(\mathrm{F}=0.59, \mathrm{p}=0.56)$ which is similar as mentioned before in paragraph 3.2. Overall, the RMS topographies are more similar between conditions after EMG removal as the ones prior to removal of the EMG activity (cf. figure 5 and figure 3 ). The principal difference is evident as a strong decrease of the RMS values in the Move condition. In general the P300 amplitudes at Pz decreased slightly after removing the EMG activity. The repeated measures ANOVA recording (Still, Pedal, Move) $\times$ tone (Target, NonTarget) revealed a significant main effect for recording $\mathrm{F}(2,14)=3.93, \mathrm{p}=<.05$, reflecting significantly larger amplitudes for the Still (mean = $6.4 \mu \mathrm{V}, \mathrm{t} 14=4.08, \mathrm{p}<.01)$ and Pedal (mean $=5.9 \mu \mathrm{V}$, $(\mathrm{t} 14=2.84, \mathrm{p}<.05))$ condition compared to the Move condition $($ mean $=4.1 \mu \mathrm{V})$. The difference between the Pedal and Still condition failed to reach significance $(\mathrm{t} 14=1.09, \mathrm{p}=.29)$. These differences in P300 are similar to the ones reported in paragraph 3.1 without control for muscle artifacts.

The grand average accuracies $( \pm \mathrm{SD})$ on single trial level after EMG correction are $75.5( \pm 8.2), 70.0$ $( \pm 10.4)$ and $66.2 \%( \pm 8.4)$ for the Still, Pedal and Move condition respectively. These differences were deemed significant $(\mathrm{F}=4.45, \mathrm{p}<.05)$. The difference between Pedal and Still conditions was deemed significant $(\mathrm{t} 14=2.47, \mathrm{p}=<.05)$ and the accuracies in the Move condition were significantly lower when compared to the Still (t14=4.82, $\mathrm{p}<.001)$ condition, but not in comparison to the Pedal condition $(\mathrm{t} 14=1.70, \mathrm{p}=0.11)$.

\subsection{User Metrics}

We determined per subject the average normalized power in the alpha band $(8-12 \mathrm{~Hz})$ for the three conditions prior to the muscle activity removal. Mean $( \pm \mathrm{SD})$ normalized alpha power for the Still, Pedal and Move conditions are respectively 18.5 $( \pm 4.2), 16.8( \pm 3.6)$ and $14.7( \pm 3.6)$. These values differ significantly $(\mathrm{F}=3.69, \mathrm{p}<.05)$ which was caused by a lower alpha power in the Move condition as compared to the Still $(\mathrm{t} 14=2.51, \mathrm{p}<.05)$ and Pedal $(\mathrm{t} 14=2.45, \mathrm{p}<.05)$ condition. The difference between the Still and Pedal conditions failed to reach significance (t14=1.37, $\mathrm{p}=.19$ ).

Average motivational scores were highest in the Move condition with a mean $8.5(\mathrm{SD} \pm 1.0)$ followed by the Pedal condition with $7.6( \pm 1.5)$ and lastly the Still condition scored a $6.2( \pm 1.5)$. These were significantly different $(\mathrm{F}=11.28, \mathrm{p}<0.001)$ and pairwise comparisons displayed a significantly higher motivation score for the Move condition compared to the other two. This indicates that the subjects enjoyed the Move condition the most. Finally, a nearly significant correlation $(\mathrm{r}=.28, \mathrm{p}=.07)$ between the number of correctly counted tones and the single-trial classification accuracy suggested that those individuals attending more to the task had better classification accuracy. Moreover, the percentage of correctly counted tones in the Still, Pedal and Move condition were very high, respectively $98.6( \pm 1.2)$, $97.5( \pm 3.3)$ and $97.7 \%( \pm 2.6)$.

\section{Discussion}

Mobile EEG allows to study the brain in real unconstrained environments. Previous studies with (mobile) EEG did not control independently for the increase in cognitive demands by the complex natural environment and the increase in physical involvement has merely been studied in simulated environments (e.g. $[29,30,31,32,33,34,35,36,51,52])$. We evaluated the ERP characteristics and classification performance of a three-class auditory oddball paradigm recorded in outdoor scenarios's while biking on either a fixed bike or freely around. The Move condition was an unconstrained ride around the place, representing a fully realistic scenario. Above chance P300 singletrial classification in these highly dynamic real life environments while biking outdoors was achieved. Certain artifact patterns were identified in the free biking condition, but we show that neither these nor the increase in movement explain the reduction in classification accuracy and P300 waveform in the real-life biking condition as compared to the control conditions. Therefore this study provides strong evidence that the higher cognitive demands 

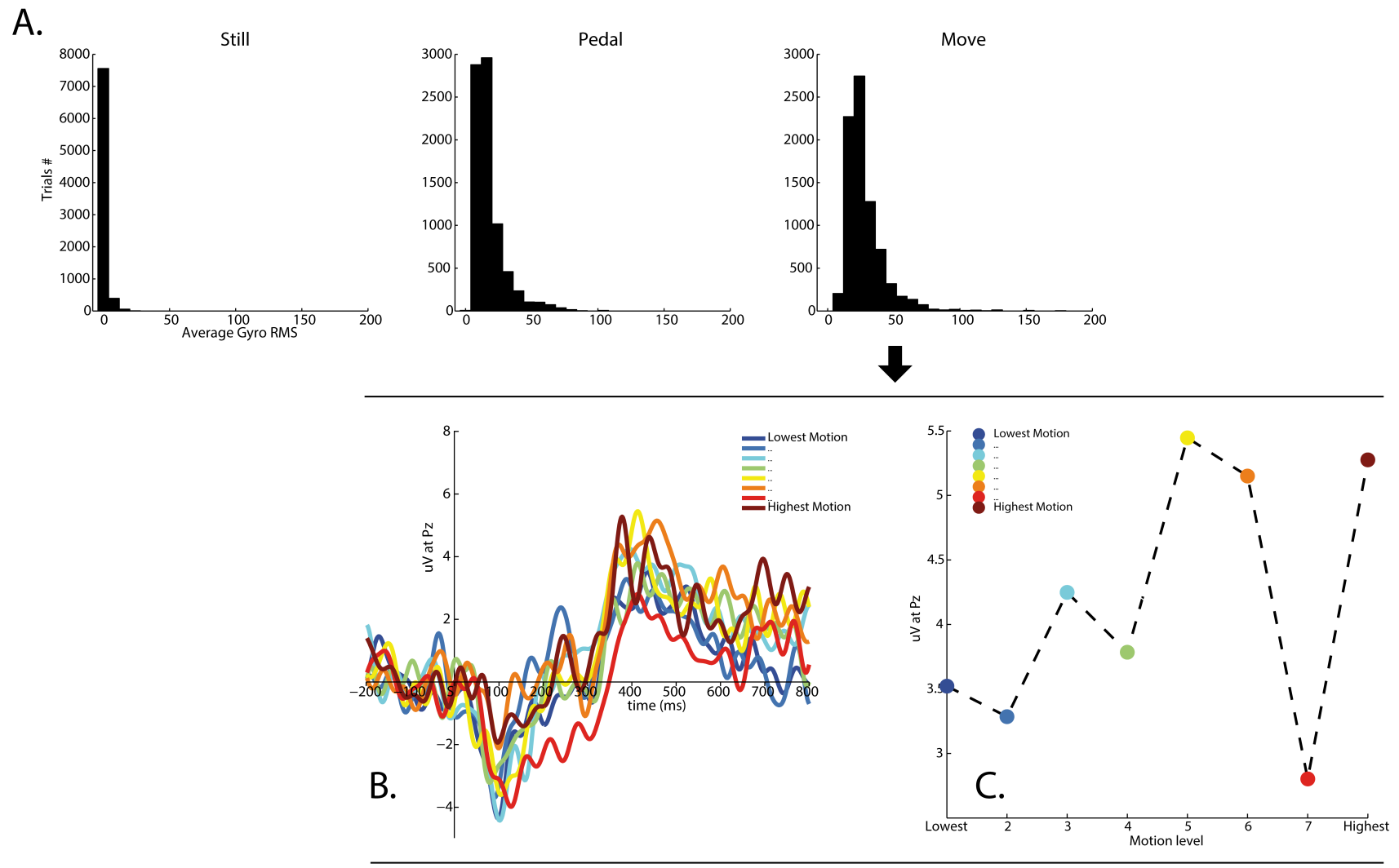

Figure 4. A. Averaged (over 3 axis) gyro RMS per trial for the Still, Pedal and Move condition from left to right respectively. B. The average ERPs at Pz to eight divisions of the Move condition data based on the gyro RMS in A(most right figure). The color indicates the degree of motion and all degrees have equal number of trials. Finally in C the peak P300 from B in contrast to the eight motion degrees; the P300 peaks seem to be unrelated to the degree of motion.
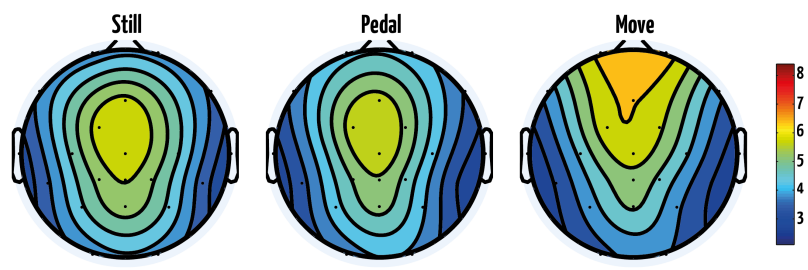

Figure 5. Median RMS values of the baseline interval [-2000]ms of the Target trials at each electrode for the Still, Pedal and Move condition after EMG removal.

are the underlying cause of the reduced attentional brain responses to auditory stimuli. This reasoning corresponds to the intuitive explanation that biking around in a natural environment requires definitely more cognitive resources as the non-mobile conditions.

On the grand average a decreased P300 peak was observed in the Move condition as compared to the other (more restricted) conditions (Figure 3, P300 topo plots). Similar effects were notable after controlling for muscle artifacts. This decrease in P300 during outdoor moving conditions was similarly described by $[8,9]$, albeit without control for artifacts and movement. Interestingly, N100 peak values for all stimulus types did not differ significantly between our conditions. We reason that the P300 and N100 should be influenced equally by physical artifacts. Since predominantly the P300 is altered between conditions this suggests that the effect of the Move condition on the P300 is larger as compared to the N100.

Additionally a mildly increased latency of the P300 was found in the Move condition, although this was not deemed significant, it may be suggestive of increased cognitive load. Indoor exercise studies suggest an increase in P300 during or after physical pedaling on a bike $[29,30,31]$. In our ERP analysis no clear distinction could be made between the Pedal and Still condition; similar P300 waveforms were observed which is similar to the results of [32]. These differences may originate from the paradigm that is used to generate a P300. The three-class auditory oddball task is dependent on the ability to attend to the acoustic stimuli and that may be more difficult with a dual task of listening and biking in a distractive environment. Another measure related to the level of cognitive functioning is the EEG power in the alpha band. A decrease of alpha power may be indicative of increases in task difficulty and mental workload $[19,53,54]$. The alpha power decrease in the move 
conditions suggests a lower focus on the auditory targets which can be related to increased difficulty due to the real-life circumstances as compared to the pedal and still conditions. An alternative explanation that we could not rule out is that the decrease in alpha power may be correlated with the extent of physical engagement.

The gyro motion quantification displayed a clear increase in head motion in the Pedal and Move conditions as compared to the Still condition (Figure 4A). However, similar ERPs were observed in the Still and Pedal condition illustrating that the wireless system used for recording and the unconstrained setup did not affect the EEG quality at channel Pz. In the real-life Move condition we split the trials to eight bins with increasing motion levels. This did not lead to a decreased P300 waveform or peak amplitude as was depicted in Figure 4B-C. Furthermore, the correlation between P300 amplitude and the head-motion was found to be minor, especially in the Move condition $(\mathrm{r}=-.16)$. A smaller P300 while biking can be alternatively explained by that the brain is occupied with extra tasks over and above the experimental task, for example the evaluation of the dynamic acoustic and visual environment and ensuring that the bike stays on the road. Similar reasoning was put forward by [9].

The EEG RMS values between conditions were only significantly higher at the frontal and posterior electrodes (Figure 3) for the Move condition. Further analysis of these channels with higher RMS values in the Move conditions showed sporadic low frequency fluctuations (head motion) and short, higher frequency range artifacts that are indicative of muscle activity. This can be attributed to a mix of electrode movement and muscle tension which is more elevated while riding a bike as opposed to the fixed conditions. Similarly there was an increase in gyro motion for the Move condition (Figure 4a). The fact that central channels such as $\mathrm{Cz}$ and $\mathrm{Pz}$ remain almost unaffected indicated that the observed artifacts did not have a large impact on the ERP results. The increase in head motion in the Move condition was expected to increase the muscle acitivity as well. This was confirmed by the large decrease of baseline RMS in the Move condition after controlling for muscle artifacts. Generally, controlling for muscle artifacts did not lead to different insights. Finally, the RMS topographies and degree of motion artifacts varied across subjects which is in line with recent views on motion artifacts and EEG [27].

Single trial characteristics have been assessed by computing rLDA classification results as is routinely done in BCI applications. For a practical BCI application, approaches that reduce the subjectspecific calibration time have gained momentum for BCI processing $[14,55,56]$. Riemannian geometry in particular has been shown to be very powerful for P300 classification and easy generalization over subjects [55]. Furthermore, the incorporation of additional structural properties present in the paradigm [14], or a reliance on stimulus presentation distributions through Bayesian statistics [56] could be beneficial. A valuable future line of research would be to see how these more profound methods would perform on real-life data such as the Move condition in the current study. A recent study on motor imagery suggested that methods which work well in a lab environment can perform poorly in realistic application scenarios [57]. The differences between conditions may be larger in real-life scenarios as compared to restricted lab setups. Differences between our conditions were also observed from the cross-session trained classifier models which performed consistently lower compared to session-specific classification (c.q. Table 1 and Table 2); when trained on the Move condition large differences in accuracy can be observed for the Still and Pedal condition. This is supported by the high average correlation $(\mathrm{r}=0.70)$ between the subject P300 waveforms of the Still and Pedal condition, but lower for comparisons of these to the Move condition, $(\mathrm{r}=0.61)$ and $(\mathrm{r}=0.54)$ respectively. The observed differences between conditions are indicative of non-stationarities or unidentified artifactual influences in the signal and transferring these session-specific differences might improve classification [58]. Furthermore higher correlations were found between the gyro motion and the rLDA accuracy as between the gyro motion and the P300, suggesting that motion artifacts (at the outer channels) might have an influence of the rLDA results as compared to the P300 in our ERP analysis.

It is vital to understand that the precise extent by which participants could focus their attention on the target tone while blocking out the distractor stimulus is unknown. Future measurements of the environment by means of video and audio monitoring may provide better control of auditory distraction events. Future analysis may also look into different types of head motions in the gyro signals. Increased left-right motion patterns may be indicative of distracting events whereas nodding patterns occur more naturally during biking and walking. Several subjects that attended to the high pitched tones reported that they sometimes confused the baseline tones with the high tones. This may be controlled by measuring the hearing thresholds prior to the experiment. However, post-hoc analysis showed no effect of tone on the classification accuracy.

Indoor EEG experiments can be very long and monotonous for the subjects as external influences and distractions are minimized. High scores on the motivation question for the Move condition illustrated 
that subjects enjoyed the real-life condition more even though the distractions made it more difficult to focus on the auditory mental task. This is a promising finding as user experience is an important factor in BCI as well [33, 59].

The current study presents several limitations to the analysis and setup. The self-reported motivation score is subject to bias and a standardized test such as used in [43] is likely to provide a more reliable measure. Subjects pedaled at a user-defined comfortable pace in the Pedal and Move conditions. There was no control of speed in our study, although in related treadmill walking studies the speed of walking was shown to be related to the number of artifacts in the EEG [20, 27]. However, forcing users to stay in a specific speed range might cause additional bias since subjects may be controlling for speed constantly. Future work that combines heart-rate monitoring with the mobile EEG may provide complementary information regarding the degree of physical exercise [30]. Moreover, heart rate has been identified as an indicator for mental state switches that could have an influence on the EEG signals [23]. Applying additional motion correction techniques may lead to new insights regarding comparisons of the classifiers. A future comparison of artifact removal techniques on real-life mobile EEG data as presented in the current study would therefore be very interesting [11, 27]. Finally users could have felt abashed as point of attention while biking outside which may have lowered the ability to focus on the auditory tones. Fortunately new hardware solutions are prominent to make the EEG equipment even less distractive and visible $[24,25]$ which is a promising development.

\section{Conclusion}

In summary, we evaluated the ERP and single trial classification characteristics of a three-class auditory oddball paradigm recorded in outdoor scenario's while biking on either a fixed bike or freely around. We identified elevated noise levels in the natural biking condition affecting only the outer channels. The influence of the natural head motion on the ERP waveforms on $\mathrm{Pz}$ was minimal and controlling for muscle artifacts reduced the overall noise level but did not lead to different insights regarding the P300 differences between conditions. A reduction in P300 in the Move condition led to lower classification accuracies obtained with rLDA. The reduction in P300 in the Move condition can be attributed to increased cognitive load due to being in a reallife scenario. These findings suggest that cognitive paradigms measured in natural real-life scenarios, such as the auditory oddball results presented here, are influenced significantly by increased cognitive load due to being in an unconstrained environment.

\section{Acknowledgments}

We are grateful to the reviewers for providing useful comments on the manuscript. Research supported by Research Council KUL: CoE PFV/10/002 (OPTEC); Belgian Federal Science Policy Office: IUAP P7/19/(DYSCO, Dynamical systems, control and optimization, 20122017), iMinds Medical Information Technologies SBO 2015; Flemish Government, FWO projects: G.0427.10N; EU: The research leading to these results has received funding from the European Research Council under the European Union's Seventh Framework Programme (FP7/2007-2013) / ERC Advanced Grant: BIOTENSORS (no. 339804). This paper reflects only the authors' views, and the Union is not liable for any use that may be made of the contained information.

\section{References}

[1] John Polich. Updating P300: an integrative theory of P3a and P3b. Clinical neurophysiology, 118(10):2128-2148, 2007.

[2] A. Furdea, S. Halder, D.J. Krusienski, D. Bross, F Nijboer, N. Birbaumer, and A. Kübler. An auditory oddball (P300) spelling system for brain-computer interfaces. Psychophysiology, 46(3):617-625, 2009.

[3] Stefan Debener, C Kranczioch, Christoph S Herrmann, and Andreas K Engel. Auditory novelty oddball allows reliable distinction of top-down and bottomup processes of attention. International Journal of Psychophysiology, 46(1):77-84, 2002.

[4] S. Halder, M. Rea, R. Andreoni, F. Nijboer, E.M. Hammer, S.C. Kleih, N. Birbaumer, and A. Kübler. An auditory oddball brain-computer interface for binary choices. Clinical Neurophysiology, 121(4):516-523, 2010.

[5] Niels Birbaumer, Nimr Ghanayim, Thilo Hinterberger, Iver Iversen, Boris Kotchoubey, Andrea Kübler, Juri Perelmouter, Edward Taub, and Herta Flor. A spelling device for the paralysed. Nature, 398(6725):297-298, 1999.

[6] Thorsten O Zander and Christian Kothe. Towards passive brain-computer interfaces: applying brain-computer interface technology to human-machine systems in general. Journal of neural engineering, 8(2):025005, 2011.

[7] Jan BF Van Erp, Fabien Lotte, and Michael Tangermann. Brain-computer interfaces: beyond medical applications. Computer, (4):26-34, 2012.

[8] Stefan Debener, Falk Minow, Reiner Emkes, Katharina Gandras, and Maarten Vos. How about taking a low-cost, small, and wireless EEG for a walk? Psychophysiology, 49(11):1617-1621, 2012.

[9] Maarten De Vos, Katharina Gandras, and Stefan Debener. Towards a truly mobile auditory brain-computer interface: Exploring the P300 to take away. International Journal of Psychophysiology, 91(1):46-53, 2014.

[10] Bin He, Todd Coleman, Guy M Genin, Gary Glover, Xiaoping $\mathrm{Hu}$, Nessa Johnson, Tianming Liu, Scott Makeig, Paul Sajda, and Kaiming Ye. Grand challenges in mapping the human brain: NSF workshop report. 
IEEE Trans. Biomed. Engineering, 60(11):2983-2992, 2013.

[11] Vojkan Mihajlovic, Bernard Grundlehner, Ruud Vullers, and Julien Penders. Wearable, wireless EEG solutions in daily life applications: what are we missing? Biomedical and Health Informatics, IEEE Journal of, 19(1):6-21, 2015.

[12] Peter Aspinall, Panagiotis Mavros, Richard Coyne, and Jenny Roe. The urban brain: analysing outdoor physical activity with mobile EEG. British journal of sports medicine, pages bjsports-2012, 2013.

[13] Maarten De Vos and Stefan Debener. Mobile EEG: towards brain activity monitoring during natural action and cognition. International journal of psychophysiology: official journal of the International Organization of Psychophysiology, 91(1):1-2, 2014.

[14] Rob Zink, Bori Hunyadi, Sabine Van Huffel, and Maarten De Vos. Tensor-based classification of auditory mobile BCI without subject-specific calibration phase. Journal of Neural Engineering, 2016.

[15] Matthieu Duvinage, Thierry Castermans, Thierry Dutoit, M Petieau, T Hoellinger, C De Saedeleer, K Seetharaman, and G Cheron. A P300-based quantitative comparison between the Emotiv Epoc headset and a medical EEG device. Biomedical Engineering, 765:2012-764, 2012.

[16] Matthieu Duvinage, Thierry Castermans, Mathieu Petieau, Thomas Hoellinger, Guy Cheron, and Thierry Dutoit. Performance of the emotiv epoc headset for P300-based applications. Biomedical engineering online, 12(1):56, 2013.

[17] Maarten De Vos, Markus Kroesen, Reiner Emkes, and Stefan Debener. P300 speller BCI with a mobile eeg system: comparison to a traditional amplifier. Journal of neural engineering, 11(3):036008, 2014.

[18] SC Kleih, T Kaufmann, C Zickler, S Halder, F Leotta, F Cincotti, F Aloise, A Riccio, C Herbert, D Mattia, et al. Out of the frying pan into the fire-the P300based BCI faces real-world challenges. Progress in brain research, 194:27-46, 2011.

[19] Trevor Thompson, Tony Steffert, Tomas Ros, Joseph Leach, and John Gruzelier. EEG applications for sport and performance. Methods, 45(4):279-288, 2008.

[20] Thierry Castermans, Matthieu Duvinage, Guy Cheron, and Thierry Dutoit. About the cortical origin of the lowdelta and high-gamma rhythms observed in EEG signals during treadmill walking. Neuroscience letters, 561:166170, 2014.

[21] Alessandro Presacco, Ronald Goodman, Larry Forrester, and Jose Luis Contreras-Vidal. Neural decoding of treadmill walking from noninvasive electroencephalographic signals. Journal of neurophysiology, 106(4):1875-1887, 2011.

[22] Siddharth Kohli and Alexander J Casson. Towards out-ofthe-lab EEG in uncontrolled environments: Feasibility study of dry EEG recordings during exercise bike riding. In Engineering in Medicine and Biology Society (EMBC), 2015 37th Annual International Conference of the IEEE, pages 1025-1028. IEEE, 2015.

[23] Tobias Vogt, Rainer Herpers, Christopher D Askew, David Scherfgen, Heiko K Strüder, and Stefan Schneider. Effects of exercise in immersive virtual environments on cortical neural oscillations and mental state. Neural plasticity, 2015, 2015.

[24] Martin G Bleichner, Micha Lundbeck, Matthias Selisky, Falk Minow, Manuela Jäger, Reiner Emkes, Stefan Debener, and Maarten De Vos. Exploring miniaturized EEG electrodes for brain-computer interfaces. an EEG you do not see? Physiological reports, 3(4):e12362, 2015.

[25] Stefan Debener, Reiner Emkes, Maarten De Vos, and
Martin Bleichner. Unobtrusive ambulatory EEG using a smartphone and flexible printed electrodes around the ear. Scientific reports, 5, 2015.

[26] Jessica Askamp and Michel JAM van Putten. Mobile EEG in epilepsy. International journal of psychophysiology, 91(1):30-35, 2014.

[27] Julia E Kline, Helen J Huang, Kristine L Snyder, and Daniel P Ferris. Isolating gait-related movement artifacts in electroencephalography during human walking. Journal of neural engineering, 12(4):046022, 2015.

[28] Laura Frolich, Irene Winkler, Klaus-Robert Muller, and Wojciech Samek. Investigating effects of different artefact types on motor imagery BCI. In Engineering in Medicine and Biology Society (EMBC), 2015 37th Annual International Conference of the IEEE, pages 1942-1945. IEEE, 2015.

[29] Yu-Kai Chang, Caterina Pesce, Yi-Te Chiang, ChengYuh Kuo, and Dong-Yang Fong. Antecedent acute cycling exercise affects attention control: an ERP study using attention network test. Frontiers in human neuroscience, 9, 2015.

[30] Ryan L Olson, Yu-Kai Chang, Christopher J Brush, Andrea N Kwok, Valentina X Gordon, and Brandon L Alderman. Neurophysiological and behavioral correlates of cognitive control during low and moderate intensity exercise. NeuroImage, 2015.

[31] Matthew B Pontifex and Charles H Hillman. Neuroelectric and behavioral indices of interference control during acute cycling. Clinical Neurophysiology, 118(3):570-580, 2007.

[32] Tobias Vogt, Rainer Herpers, David Scherfgen, Heiko K Strüder, and Stefan Schneider. Neuroelectric adaptations to cognitive processing in virtual environments: an exercise-related approach. Experimental brain research, 233(4):1321-1329, 2015

[33] Yasuo Yagi, Kerry L Coburn, Kristi M Estes, and James E Arruda. Effects of aerobic exercise and gender on visual and auditory P300, reaction time, and accuracy. European journal of applied physiology and occupational physiology, 80(5):402-408, 1999.

[34] Fabien Grego, Jean-Marc Vallier, Maya Collardeau, Stéphane Bermon, Patricia Ferrari, Mirande Candito, Pascale Bayer, Marie-Noëlle Magnié, and Jeanick Brisswalter. Effects of long duration exercise on cognitive function, blood glucose, and counterregulatory hormones in male cyclists. Neuroscience letters, 364(2):76-80, 2004.

[35] Klaus Gramann, Joseph T Gwin, Daniel P Ferris, Kelvin Oie, Tzyy-Ping Jung, Chin-Teng Lin, Lun-De Liao, and Scott Makeig. Cognition in action: imaging brain/body dynamics in mobile humans. Reviews in the Neurosciences, 22(6):593-608, 2011.

[36] Yuan-Pin Lin, Yijun Wang, and Tzyy-Ping Jung. Assessing the feasibility of online ssvep decoding in human walking using a consumer eeg headset. Journal of neuroengineering and rehabilitation, 11(1):1, 2014.

[37] Hans Hallez, Maarten De Vos, Bart Vanrumste, Peter Van Hese, Sara Assecondi, Koen Van Laere, Patrick Dupont, Wim Van Paesschen, Sabine Van Huffel, and Ignace Lemahieu. Removing muscle and eye artifacts using blind source separation techniques in ictal eeg source imaging. Clinical Neurophysiology, 120(7):12621272, 2009.

[38] Pedro MR Reis, Felix Hebenstreit, Florian Gabsteiger, Vinzenz von Tscharner, and Matthias Lochmann. Methodological aspects of eeg and body dynamics measurements during motion. Towards a New Cognitive Neuroscience: Modeling Natural Brain Dynamics, page 9, 2014.

[39] Heike Leutheuser, Florian Gabsteiger, Felix Hebenstreit, 
Pedro Reis, Matthias Lochmann, and Bjoern Eskofier. Comparison of the amica and the infomax algorithm for the reduction of electromyogenic artifacts in eeg data. In Engineering in Medicine and Biology Society (EMBC), 2013 35th Annual International Conference of the IEEE, pages 6804-6807. IEEE, 2013.

[40] Wim De Clercq, Anneleen Vergult, Bart Vanrumste, Wim Van Paesschen, and Sabine Van Huffel. Canonical correlation analysis applied to remove muscle artifacts from the electroencephalogram. Biomedical Engineering, IEEE Transactions on, 53(12):2583-2587, 2006.

[41] De Maarten Vos, Stephanie Riès, Katrien Vanderperren, Bart Vanrumste, Francois-Xavier Alario, Van Sabine Huffel, and Boris Burle. Removal of muscle artifacts from eeg recordings of spoken language production. Neuroinformatics, 8(2):135-150, 2010.

[42] Yann Renard, Fabien Lotte, Guillaume Gibert, Marco Congedo, Emmanuel Maby, Vincent Delannoy, Olivier Bertrand, and Anatole Lécuyer. OpenViBE: an opensource software platform to design, test, and use braincomputer interfaces in real and virtual environments. Presence: teleoperators and virtual environments, 19(1):35-53, 2010.

[43] E Baykara, CA Ruf, C Fioravanti, I Käthner, N Simon, SC Kleih, A Kübler, and S Halder. Effects of training and motivation on auditory P300 brain-computer interface performance. Clinical Neurophysiology, 2015.

[44] Arnaud Delorme and Scott Makeig. EEGLAB: an open source toolbox for analysis of single-trial eeg dynamics including independent component analysis. Journal of neuroscience methods, 134(1):9-21, 2004.

[45] Christian Andreas Kothe and Scott Makeig. BCILAB: a platform for brain-computer interface development. Journal of neural engineering, 10(5):056014, 2013.

[46] Maarten De Vos, Jeremy D Thorne, Galit Yovel, and Stefan Debener. Let's face it, from trial to trial: comparing procedures for N170 single-trial estimation. Neuroimage, 63(3):1196-1202, 2012.

[47] R. Zink, B. Hunyadi, S. Van Huffel, and M. De Vos. Classifying the auditory P300 using mobile EEG recordings without calibration phase. In Engineering in Medicine and Biology Society (EMBC), 2015 37th Annual International Conference of the IEEE, pages 1777-1780, Aug 2015.

[48] Benjamin Blankertz, Steven Lemm, Matthias Treder, Stefan Haufe, and Klaus-Robert Müller. Single-trial analysis and classification of ERP componentsa tutorial. NeuroImage, 56(2):814-825, 2011.

[49] Fabien Lotte, Marco Congedo, Anatole Lécuyer, and Fabrice Lamarche. A review of classification algorithms for EEG-based brain-computer interfaces. Journal of neural engineering, 4, 2007.

[50] Fabien Lotte, Laurent Bougrain, and Maureen Clerc. Electroencephalography (EEG)-based brain-computer interfaces. Wiley Encyclopedia of Electrical and Electronics Engineering, 2015.

[51] Joseph T Gwin, Klaus Gramann, Scott Makeig, and Daniel P Ferris. Removal of movement artifact from high-density eeg recorded during walking and running. Journal of neurophysiology, 103(6):3526-3534, 2010.

[52] Scott E Kerick, Kelvin S Oie, and Kaleb McDowell. Assessment of eeg signal quality in motion environments. Technical report, DTIC Document, 2009.

[53] Michael E Smith, Alan Gevins, Halle Brown, Arati Karnik, and Robert Du. Monitoring task loading with multivariate eeg measures during complex forms of human-computer interaction. Human Factors: The Journal of the Human Factors and Ergonomics Society, 43(3):366-380, 2001.

[54] Wolfgang Klimesch. Eeg alpha and theta oscillations reflect cognitive and memory performance: a review and analysis. Brain research reviews, 29(2):169-195, 1999.

[55] Alexandre Barachant and Marco Congedo. A plug\&play P300 BCI using information geometry. arXiv preprint arXiv:1409.0107, 2014.

[56] Pieter-Jan Kindermans, Martijn Schreuder, Benjamin Schrauwen, Klaus-Robert Müller, and Michael Tangermann. True zero-training brain-computer interfacing-an online study. 2014.

[57] Stephanie Brandl, Johannes Hohne, Klaus-Robert Muller, and Wojciech Samek. Bringing BCI into everyday life: Motor imagery in a pseudo realistic environment. In Neural Engineering (NER), 2015 7th International IEEE/EMBS Conference on, pages 224-227. IEEE, 2015.

[58] Wojciech Samek, Frank C Meinecke, and Klaus-Robert Muller. Transferring subspaces between subjects in brain-computer interfacing. Biomedical Engineering, IEEE Transactions on, 60(8):2289-2298, 2013.

[59] Andrew Myrden and Tom Chau. Effects of user mental state on EEG-BCI performance. Frontiers in Human Neuroscience, 9:308, 2015. 